FOLIA

Amazónica

Revista del Instituto de Investigaciones

de la Amazonía Peruana

\title{
COMPORTAMIENTO AGRONÓMICO DE Oryza sativa L. (ARROZ) Y Manihot esculenta Crantz (YUCA) EN DOS LOCALIDADES DE SIEMBRA EN LORETO, PERÚ
}

\author{
Elías CUMARI LAULATE ${ }^{1}$, Juan Imerio URRELO CORREA ${ }^{1}$, \\ Rodrigo GONZALES VEGA², Julio PINEDO JIMENEZ ${ }^{1}$, \\ Joel VÁSQUEZ BARDALES ${ }^{3}$
}

1 Universidad Nacional de la Amazonía Peruana (UNAP). Facultad de Agronomía. Iquitos, Loreto, Perú.

2 Instituto Nacional de Investigación Agraria (INIA). Estación Experimental Agraria San Roque. Loreto, Perú.

3 Instituto de Investigaciones de la Amazonía Peruana (IIAP). Iquitos, Perú.

* Correo electrónico: elias.cum.lau@gmail.com

\section{RESUMEN}

En la región Loreto, se han realizado muchos esfuerzos para alcanzar altos rendimientos de la yuca y el arroz, los cuales poseen moderados potenciales de rendimiento; siendo estos cultivos, una garantía en la alimentación de la población, ya que son de mucha demanda por la población rural amazónica. El objetivo del estudio fue determinar el comportamiento de Oryza sativa L. variedad "INIA 509 la esperanza" y Manihot esculenta Crantz variedad "señorita" en dos localidades de siembra en la región Loreto. Fueron sembradas bajo un sistema de Bactris gasipaes H.B.K "pijuayo" en las localidades El Dorado y el Bolloquito ubicadas en el eje de la carretera Iquitos-Nauta en la Amazonía peruana. El cultivo del arroz mostró significancia estadística $(\mathrm{p}<0.01)$ en las características agronómicas con mayores promedios en El Dorado para altura de planta $(81,61 \mathrm{~cm})$, cantidad de matas $(21,55)$, cantidad de panojas $(21,55)$, peso de granos por mata $(0,08 \mathrm{~kg})$ y rendimiento de grano $(2656,14$ $\mathrm{k} / \mathrm{ha})$. En el cultivo de la yuca mostraron significancia estadística $(\mathrm{p}<0.01)$ en las características agronómicas con mayores promedios en el Bolloquito como altura de planta $(255,55 \mathrm{~cm})$ y altura de la primera ramificación $(130,22 \mathrm{~cm})$, mientras en El Dorado mostraron significancia estadística $(\mathrm{p}<0,05)$ para peso 
de raíz por planta $(2,59 \mathrm{~kg})$ y rendimiento de raíces $(19689,07 \mathrm{~kg} / \mathrm{ha})$, los cultivos se comportan según las localidades de siembra, expresando mejores características agronómicas y rendimientos de manera específica.

PALABRAS CLAVE: características ambientales, manejo agronómico, rendimiento, sistema de producción agroforestal.

\title{
AGRONOMIC BEHAVIOR OF Oryza sativa L. (RICE) AND Manihot esculenta Crantz (YUCA) IN TWO SEEDING LOCATIONS IN LORETO, PERU
}

\begin{abstract}
In Loreto region, many efforts have been made to obtain high yields of cassava and rice, which have moderate yield potentials; these crops are a guarantee of food for the population, since they are in great demand by the rural population of the Amazon. The objective of the study was to determine the behavior of Oryza sativa L. variety "INIA 509 la esperanza" and Manihot esculenta Crantz variety "señorita" in two localities of sowing in Loreto region. They were sowed under a system of Bactris gasipaes H.B.K "pijuayo" in the localities El Dorado and Bolloquito located in the axis of the highway Iquitos-Nauta in the Peruvian Amazon. The rice cultivation showed statistical significance $(p<0.01)$ in the agronomic characteristics with higher averages in El Dorado for plant height $(81.61 \mathrm{~cm})$, quantity of bushes (21.55), quantity of panojas (21.55), weight of grains per bush (0.08 kg) and grain yield (2656.14 k/ha). In cassava cultivation they showed statistical significance $(\mathrm{p}<0,01)$ in the agronomic characteristics with higher averages in Bolloquito as plant height $(255.55 \mathrm{~cm})$ and first branch height $(130.22 \mathrm{~cm})$, while in El Dorado they showed statistical significance $(\mathrm{p}<0.05)$ for root weight per plant $(2.59 \mathrm{~kg})$ and root yield $(19689.07 \mathrm{~kg} / \mathrm{ha})$, the crops behave according to the sowing localities, expressing better agronomic characteristics and yields in a specific way.
\end{abstract}

KEYWORDS: agroforestry production system, agronomic management, environmental characteristics, yield. 


\section{INTRODUCCIÓN}

Los cultivos tropicales de importancia en el trópico tales como la yuca, el arroz, el banano y el plátano poseen moderados potenciales de rendimiento, representando una garantía en la alimentación de la población; siendo de mucha demanda por la población rural, debido a ello vienen realizado muchos esfuerzos para alcanzar mejores rendimientos (Suárez \& Mederos, 2011). La yuca es un alimento básico para muchas familias campesinas de escasos recursos así como para la producción de peces, la ganadería y otras actividades productivas, ha constituido un valioso alimento desde la época de los aborígenes, mucho antes de la llegada de los españoles formando parte del surtido de raíces y tubérculos (Suárez \& Mederos, 2011; Contreras, 2019), el arroz es sin duda el cereal con mayor posibilidad de suplir un déficit de producción agrícola para la alimentación humana por sus cualidades nutritivas (Franquet \& Borrás, 2004), aunque pueda desarrollarse en diversos tipos de suelos; su mejor desarrollo ocurre en suelos inundables (Noches, 2019). Sin embargo estos buenos rendimientos de sus suelos aluviales no garantizan la rentabilidad en todos sus cultivos y sistemas (Labarta et al., 2007). La mayoría de los agricultores en los trópicos húmedos practican la agricultura migratoria y cambian fácilmente a sistemas de producción continua y los sistemas intermedios de tecnologías de bajo costo y bajos insumos podría ser útil como un primer paso para un uso más permanente de la tierra (Sánchez \& Benítez, 1987; Fernández \& Serrao, 1992). Las familias amazónicas establecidas en suelos de altura diversifican sus cultivos para subsistir y obtener ingresos económicos, debido a que las especies alimenticias en asociaciones tienen un mejor comportamiento que sembradas en monocultivos como se realiza en suelos del bajo (Chibnik, 1994). En fincas de pequeños agricultores predominan las mezclas de cultivos de ciclo corto, temporal y permanente; reflejando una combinación de especies en el tiempo y en el espacio, tratando de simular a la naturaleza (Trouse, 1975). Estos sistemas presentan ciertas ventajas sobre los monocultivos, sean anuales o perennes, por el uso intensivo de la tierra y mayor eficiencia del trabajo (Nair \& Fernández, 1983; Raintree \& Warner, 1986; Arévalo, 1994). Estudios realizados del arroz en Yurimaguas (Loreto), sembrados en callejón asociado a Erythrina sp. e Inga edulis (guaba) encontraron rendimientos de 1,39 y 1,24 t/ha, respectivamente (Arévalo \& López, 1996). Por su parte Zuta (1985) evaluó una variedad de Manihot esculenta Crantz (yuca) y obtuvo rendimiento de 9,7 a 17,9 t/ha de raíces. La yuca se caracteriza por su capacidad de producir en suelos ácidos y pobres (Cárdenas, 1999) y es considerada la mejor fuente de energía en sistemas agroforestales (Martínez et al., 2006).

Considerando las afirmaciones y evidencias anteriores, se ha realizado la investigación del comportamiento de variedades de Oryza sativa L. (arroz) y Manihot esculenta Crantz (yuca), sembradas de manera secuencial en parcelas establecidas de Bactris gasipaes H.B.K., a fin de buscar mayor eficiencia en el uso de los suelos y una actividad agrícola más estable. En este sentido, el presente trabajo tuvo como objetivo determinar el comportamiento de Oryza sativa L. variedad "INIA 509 la esperanza" y Manihot esculenta Crantz variedad "señorita" en dos localidades de siembra en la región Loreto.

\section{MATERIAL Y MÉTODOS}

\section{ÁREA DE ESTUDIO}

La presente investigación se ejecutó de febrero 2017 a mayo del 2018 en dos localidades. El Dorado ubicado aproximadamente en el km 25,5, campo experimental del Instituto Nacional de 


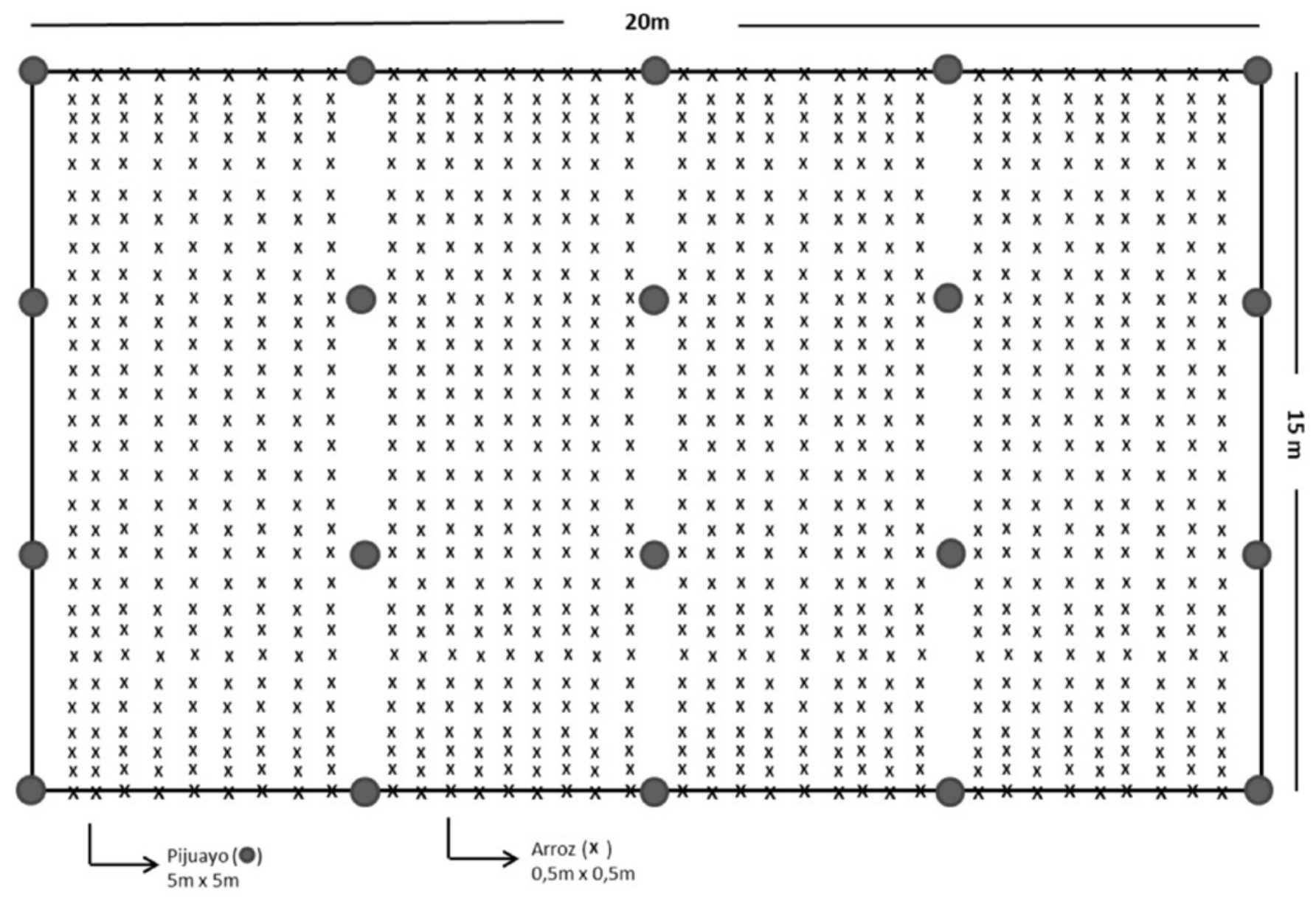

Figura 1. Croquis de la parcela experimental de la asociación pijuayo-arroz en parcelas experimentales instaladas en dos localidades de siembra en la región Loreto.

Innovación Agraria (0357'56" S. 73²4'43” 0. Altitud de $125 \mathrm{msnm}$ ), en el distrito de San Juan Bautista, provincia Maynas, departamento Loreto (Perú) y la localidad El Bolloquito ubicado en el $\mathrm{km} 76,5$ de la carretera Iquitos-Nauta, propiedad del Sr. Jorge Huamán Fachín (04²2' 21" S.; $73^{\circ}$ 33' 44" O. Altitud de $126 \mathrm{msnm}$ ), en el distrito de Nauta, provincia Loreto, departamento Loreto (Perú). El análisis de las muestras de suelos se realizó en el laboratorio de análisis de suelos, plantas y abonos de la Estación Experimental Agraria Pucallpa (INIA). Las zonas de estudio se encuentran clasificadas como bosque húmedo tropical, caracterizado por sus altas temperaturas, superiores a $26{ }^{\circ} \mathrm{C}$ y fuertes precipitaciones que oscilan entre 2000 mm y 4000 mm por año según Holdridge (1987).

\section{PROPIEDADES QUIIMICAS DEL SUELO DE LAS LOCALIDADES DE SIEMBRA}

En la localidad de siembra El Dorado, el suelo presenta textura arcillosa (A 11\%, Ar 52\% Li $37 \%$ ), bajo contenido de materia orgánica (M.O.) 2,0 \%, bajo en fosforo con 2,03 ppm, muy bajo en potasio con $0,17 \mathrm{Cmol} / \mathrm{L}, \mathrm{pH}$ extremadamente acido 4,12 y Capacidad de intercambio catiónico efectivo (CICE) medio de 14,66.

En la localidad Bolloquito, el suelo presentó textura arcillosa (A $43 \%$, Ar $40 \%$, Li $17 \%$ ), bajo contenido de M.O. (1,74\%), bajo en fosforo $(0,76$ 
ppm), bajo en potasio $(0,04 \mathrm{Cmol} / \mathrm{Lt}), \mathrm{pH}$ del suelo extremadamente ácido $(3,97)$ y CICE bajo $(5,88)$.

\section{DISEÑO EXPERIMENTAL}

Se utilizó el diseño experimental de Bloques Completos al Azar; las variedades de arroz y yuca fueron sembradas en parcelas experimentales instaladas en dos localidades de siembra, con 15 repeticiones en cada localidad para cada variedad. El área experimental de la localidad fue de 6600 $\mathrm{m}^{2}$, cada unidad experimental fue de $300 \mathrm{~m}^{2}$, con un total de 16200 matas de arroz (1080 por parcela) (Figura 1) y 3600 plantas de yuca (240 por parcela) (Figura 2). La densidad de siembra para el arroz fue de $0,50 \times 0,50 \mathrm{~m}$, en 36 hileras por parcela (30 matas por hilera) y de la yuca de $1,0 \times 1,0 \mathrm{~m}$, en 16 hileras por parcela (15 plantas por hilera).

\section{MANEJO AGRONÓMICO}

En el campo experimental diseñado dentro de un cultivo de Bactris gasipaes H.B.K. "pijuayo", se preparó el terreno con labores de desmonte y limpieza, con la delimitación de las parcelas; primero se sembró el pijuayo con una densidad de $5 \mathrm{~m} \times 5 \mathrm{~m}$; entre esta plantación se sembró seguidamente el arroz (Figura 3A). Después de

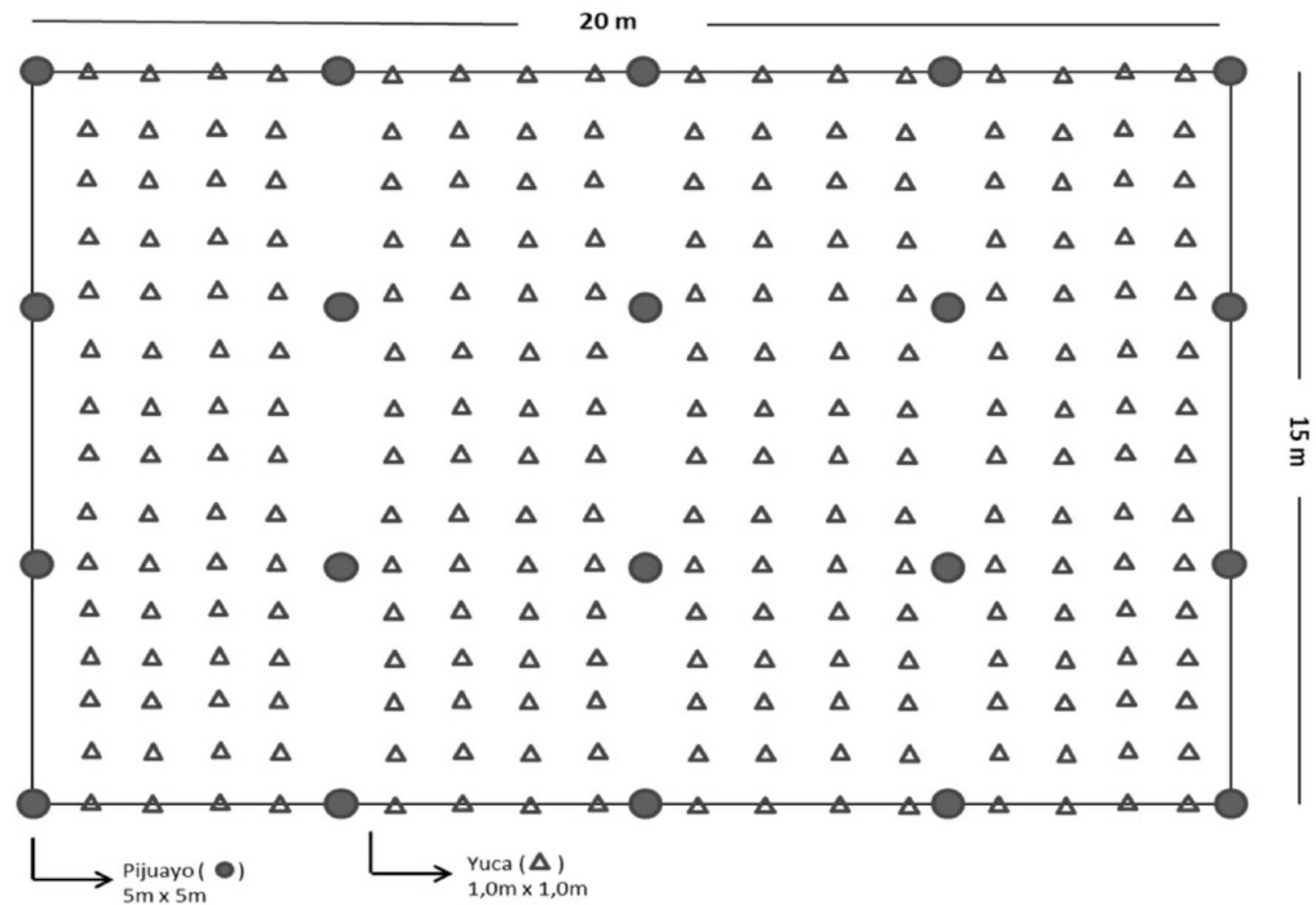

Figura 2. Croquis de la parcela experimental de la asociación pijuayo-yuca en parcelas experimentales instaladas en dos localidades de siembra en la región Loreto. 
la cosecha del arroz, se sembró la yuca (Figura 3B). En ambos cultivos se realizaron deshierbes manuales, control fitosanitario preventivo en el cultivo de arroz con insecticida ROTEBIOL con dosis de $15 \mathrm{ml}$ en 20 litros de agua más el adherente PEGASOL, más no fue necesario el control fitosanitario para el cultivo de yuca. Para corregir deficiencia de nutrientes en ambos cultivos, principalmente nitrógeno, se aplicó el abono foliar BAYFOLAN (N.F.L) 11-8-6, con una dosis de $15 \mathrm{ml}$ en 20 litros de agua. La cosecha del arroz se realizó a los 141 días después de la siembra (Figura 3C) y la cosecha de la yuca a los 260 días después de la siembra (Figura 3D). El cultivo de pijuayo alcanzó un periodo vegetativo de 1,4 años a la cosecha de la yuca, logrando una altura promedio de 1,80 m (Figura 4).

\section{EVALUACIONES DE LAS CARACTERÍSTICAS AGRONÓMICAS EN PLANTAS CULTIVADAS}

Cultivo de arroz: todas las variables se registraron a la cosecha (141 días después de la siembra), cuando el 95 a $98 \%$ de las espigas presentaron color marrón pajizo: Altura de planta
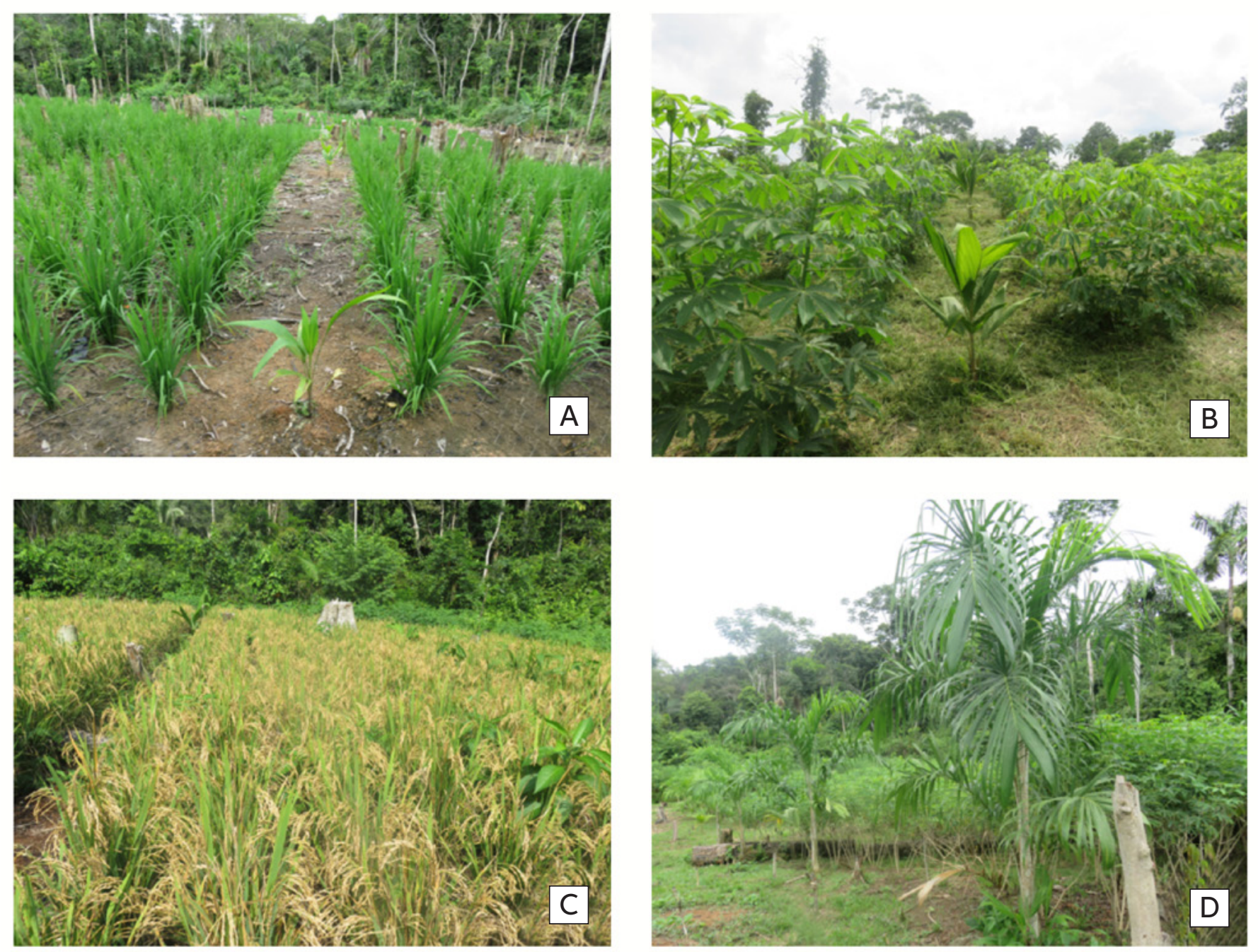

Figura 3. A) Asociación pijuayo- arroz, B). Asociación pijuayo- yuca, C) Asociación pijuayo-arroz previa a la cosecha del arroz y D) Asociación pijuayo-yuca previa a la cosecha de la yuca. 


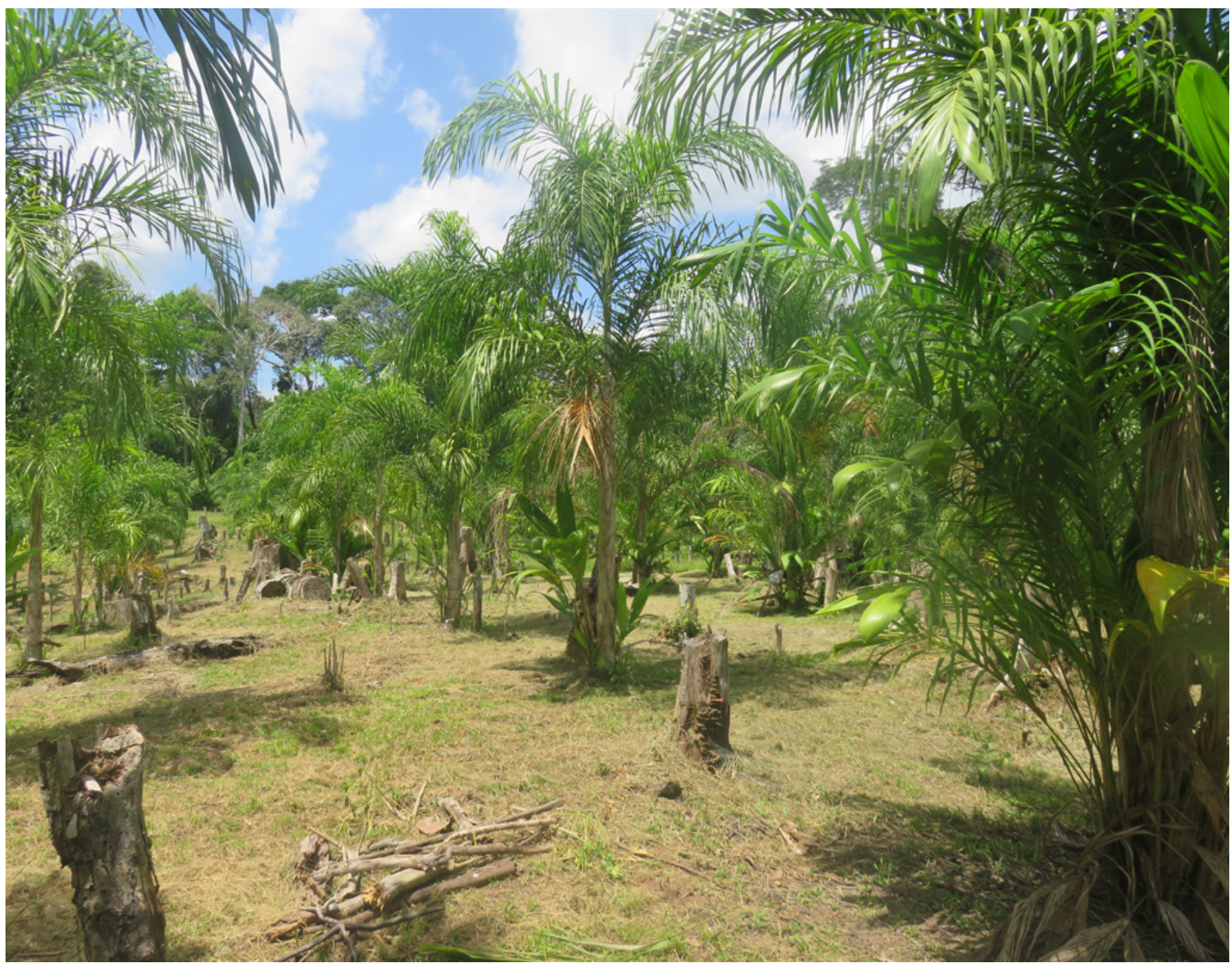

Figura 4. Plantación en monocultivo de pijuayo al final del experimento.

$(A P)$, con mediciones desde la superficie del suelo hasta la panícula más alta. Número de macollos (NM), mediante el conteo de macollos. Número de panojas (NP), mediante el conteo de panojas. Peso de granos por mata (PGM), se pesó los granos llenos y libres de impurezas. El rendimiento de granos por parcela se registró del total de plantas, asumiendo una humedad de grano del $14 \%$.

Cultivo de yuca: todas las variables se registraron a la cosecha (260 días después de la siembra). Altura de Planta (AP), tomado desde la superficie del suelo hasta la parte más alta. Altura primera ramificación (APR), tomados desde la superficie del suelo hasta la primera bifurcación. Diámetro del tallo (DT), se tomó a $20 \mathrm{~cm}$ del tallo sobre la superficie del suelo con el uso de Vernier. Número de raíces por planta (NRP), conteo del total de raíces tuberosas. Longitud de raíz (LR), se tomó desde la inserción de tallo. Diámetro de la raíz $(D R)$, se tomó de la parte central de las raíces tuberosas. Peso de raíz por planta (PRP), tomado del total de raíces tuberosas; se usó una balanza de precisión. Rendimiento de raíz por parcela (PRP), tomados del total de plantas. 


\section{ANÁLISIS ESTADÍSTICO}

Se analizó mediante la prueba de normalidad de Shapiro-Wilks modificado y la prueba de homogeneidad de varianzas de Fisher. Se empleó la prueba de comparaciones independientes $\mathrm{T}$ de Student para los datos que presentaron distribución normal y la prueba U de MannWhitney para los datos que no presentaron distribución normal, se empleó el software Infostat versión 2018.

\section{RESULTADOS Y DISCUSIÓN}

En el cultivo de arroz se muestra que hay efecto significado $(\mathrm{p}<0,01)$ de localidad de siembra sobre el númeroro de macollos (NM), número de panojas (NP), altura de planta (AP), peso de granos por mata (PGM) y rendimiento de granos por parcela (RGP). El Dorado presenta condiciones más favorables para el cultivo con una diferencia (4,68 en NM, 8,75 en NP, 16,49 en AP, 0,04 en GM y 1274,46 en RGP), asumimos que las condiciones químicas y físicas del suelo de ésta localidad al presentar mayor concentración de M.O., P, K y pH, estarían mejorando la cantidad de macollos $(21,5)$, cantidad de panoja $(21,5)$, altura de planta $(81,61 \mathrm{~cm})$, peso de granos por mata $(0,08 \mathrm{~kg})$ y rendimiento de grano por parcela $(2656,14 \mathrm{~kg})$, ligeramente inferior a los 2 $930 \mathrm{~kg} / \mathrm{ha} /$ Campaña de 2019 en la región Loreto en zonas inundables (INIA, 2019). El pH del suelo favorece el crecimiento del arroz, aunque pueda desarrollarse en una gran variedad de suelos. Sin embargo, esta planta al ser originaria de suelos cercanos a ríos, su crecimiento es mejor en suelos de textura fina y media (arcillas) con gran cantidad de material orgánico con un $\mathrm{pH}$

Tabla 1. Prueba T de Student para características agronómicas de Oryza sativa L. variedad "INIA 509 La Esperanza” con distribución normal, en dos localidades en la región Loreto.

\begin{tabular}{cccccccc}
\hline Variable & Localidad & $\mathbf{n}$ & Media & DE & Varianza & T & p (2 colas) \\
\hline \multirow{2}{*}{$\begin{array}{c}\text { Cantidad de macollos } \\
\text { (Unidad) }\end{array}$} & Bolloquito & 15 & 16,87 & 2,72 & 7,41 & $-5,58$ & 0,0001 \\
\cline { 2 - 7 } & El Dorado & 15 & 21,55 & 1,77 & 3,14 & & \\
\hline \multirow{2}{*}{$\begin{array}{c}\text { Cantidad de panojas } \\
\text { (Unidades) }\end{array}$} & Bolloquito & 15 & 12,80 & 1,93 & 3,74 & $-6,58$ & 0,0001 \\
\cline { 2 - 7 } & El Dorado & 15 & 21,55 & 1,77 & 3,14 & & \\
\hline
\end{tabular}

Tabla 2. Prueba U de Mann-Whitney para características agronómicas de Oryza sativa L. variedad "INIA 509 La Esperanza" sin distribución normal, en dos localidades en la región Loreto.

\begin{tabular}{cccccccc}
\hline Variable & Localidad & $\mathbf{n}$ & Media & DE & Mediana & W & p(2 colas) \\
\hline Altura de planta $(\mathrm{cm})$ & Bolloquito & 15 & 65,12 & 6,08 & 64 & 147 & 0,0004 \\
\cline { 2 - 7 } & El Dorado & 15 & 81,61 & 12,24 & 82 & & \\
\hline $\begin{array}{c}\text { Peso granos por mata } \\
(\mathrm{kg})\end{array}$ & Bolloquito & 15 & 0,04 & 0,01 & 0,04 & 0.120 & 0,0001 \\
\cline { 2 - 7 } & El Dorado & 15 & 0,08 & 0,01 & 0,08 & & \\
\hline $\begin{array}{c}\text { Rendimiento de } \\
\text { granos }(\mathrm{kg} / \mathrm{ha})\end{array}$ & Bolloquito & 15 & 1381,68 & 258,79 & 1368,0 & 120 & 0,0001 \\
\cline { 2 - 7 } & El Dorado & 15 & 2656,14 & 455,37 & 2599,2 & & \\
\hline
\end{tabular}


Tabla 3. Prueba U de Mann-Whitney para características agronómicas de Manihot esculenta Crantz variedad "Señorita", en dos localidades en la región Loreto.

\begin{tabular}{cccccccc}
\hline Variable & Localidad & N & Media & DE & Mediana & W & p(2 colas) \\
\hline \multirow{2}{*}{ Altura planta (AP) } & Bolloquito & 15 & 255,55 & 35,86 & 261 & 297 & 0,0075 \\
\cline { 2 - 8 } & El Dorado & 15 & 218,65 & 23,22 & 212 & & \\
\hline
\end{tabular}

Tabla 4. Prueba T de Student para características agronómicas de Manihot esculenta Crantz variedad "Señorita", en dos localidades en la región Loreto.

\begin{tabular}{|c|c|c|c|c|c|c|c|}
\hline Variable & Localidad & $\mathrm{N}$ & Media & DE & Varianza & $\mathrm{T}$ & $\mathrm{p}$ ( 2 colas $)$ \\
\hline \multirow{2}{*}{$\begin{array}{l}\text { Altura primera } \\
\text { ramificación (cm) }\end{array}$} & Bolloquito & 15 & 130,22 & 25,31 & 640,76 & 3,31 & 0,0026 \\
\hline & El Dorado & 15 & 100,28 & 24,2 & 585,80 & & \\
\hline \multirow{2}{*}{ Diámetro de tallo (cm) } & Bolloquito & 15 & 2,23 & 0,27 & 0,07 & 0,64 & 0,5278 \\
\hline & El Dorado & 15 & 2,17 & 0,27 & 0,07 & & \\
\hline \multirow{2}{*}{$\begin{array}{l}\text { No de raíces por planta } \\
\text { (Unidad) }\end{array}$} & Bolloquito & 15 & 3,01 & 0,63 & 0,39 & $-0,86$ & 0,3959 \\
\hline & El Dorado & 15 & 3,29 & 0,67 & 0,45 & & \\
\hline \multirow{2}{*}{ Longitud de raíz (cm) } & Bolloquito & 15 & 27,62 & 2,29 & 5,25 & $-1,23$ & 0,2290 \\
\hline & El Dorado & 15 & 28,47 & 1,43 & 2,05 & & \\
\hline \multirow{2}{*}{ Diámetro de raíz (cm) } & Bolloquito & 15 & 5,67 & 0,39 & 0,15 & $-0,90$ & 0,3770 \\
\hline & El Dorado & 15 & 5,81 & 0,45 & 0,20 & & \\
\hline \multirow{2}{*}{$\begin{array}{l}\text { Peso de raíz por planta } \\
(\mathrm{Kg})\end{array}$} & Bolloquito & 15 & 1,96 & 0,63 & 0,39 & $-2,22$ & 0,0349 \\
\hline & El Dorado & 15 & 2,59 & 0,91 & 0,83 & & \\
\hline \multirow{2}{*}{$\begin{array}{l}\text { Rendimiento de raíces } \\
\qquad(\mathrm{kg} / \mathrm{ha})\end{array}$} & Bolloquito & 15 & 14873,60 & 0,63 & 22628209,83 & $-2,22$ & 0,0349 \\
\hline & El Dorado & 15 & 19689,07 & 0,91 & 48151596,50 & & \\
\hline
\end{tabular}

que oscila entre 6 a 7, que proporciona una gran cantidad de nutrientes a la planta, solo que es más laborioso y existe un alto riesgo de pérdida debido a la dinámica de la inundación (Tamhane et al., 1978; List et al., 2019; Noches, 2019).

En el cultivo de yuca, se muestra que hay efecto significativo $(\mathrm{p}<0,01)$ de localidad de siembra sobre altura de planta (AP) y altura de la primera ramificación (APR), siendo en la localidad el Bolloquito mayores promedios para ambas variables $(\mathrm{AP}=255,5 \mathrm{~cm}$; $A P R=130,22 \mathrm{~cm})$ éste comportamiento con un promedio mayor con respecto a la localidad de El Dorado está influenciado por las mejores condiciones de suelo que favorece un mayor desarrollo en altura de planta, siendo una variable cuantitativa que tiene mayor predominio en el comportamiento según las condiciones edafológicas de la localidad de 
siembra. Sin embargo, a pesar que las condiciones ecológicas son iguales, las características edafológicas de cada parcela, son el factor que más afecta la expresión del cultivo (Ortiz et al., 2004). Con respecto al diámetro de tallo (DT), cantidad de raíces por planta (NRP), longitud de raíz (LR) y diámetro de raíz (DR) no muestran diferencias estadísticas significativas $(p>0,05)$ en relación a la localidad de siembra, estas características estarían comportándose de manera indiferente a las condiciones de suelo, características que muestran menor influencia de las condiciones edáficas, muchas plantas que se han introducido desde áreas geográficas muy distantes a las nativas fueron particularmente favorecidas por las perturbaciones causadas por la actividad agrícola propiciando su buen desarrollo (Blanco, 2016). El peso de la raíz, está determinado por su longitud, diámetro y cantidad, las cuales se comportaron discretamente a las localidades de siembra con condiciones edáficas particulares; sin embargo, al presentar valores numéricamente superiores en la localidad de El Dorado, nos permite asumir que el peso de raíz al estar asociadas a estas características y a promedios ligeramente mayores en la localidad de siembra, el peso de raíces muestra diferencias significativas $(\mathrm{p}<0,05)$ con un mayor peso de raíz por planta de 2,59 kg y el rendimiento de raíces de 19 689,07 $\mathrm{kg} / \mathrm{ha}$, deduciendo que las localidades de siembra y de las variedades utilizadas determinan el comportamiento agronómico de los cultivos (Pérez-Crespo, 1991). Las condiciones edáficas están asociadas a un control oportuno de las malezas que favoreció un mejor comportamiento de estos cultivos, tal apreciación lo menciona De Datta (1986) indicando que en zonas tropicales las malezas reducen los rendimientos del arroz, debido a la competencia por luz, espacio, agua y nutrientes; y además son hospederos de plagas y enfermedades. Páez et al. (1992) precisa que las malezas poseen una capacidad de competencia debido a su facilidad de adaptación a los diversos medios y a condiciones similares a las del arroz causando mayores daños al cultivo. De igual modo estas prácticas favorecieron al rendimiento de yuca, al respecto Scharff (1988), en su investigación sobre el efecto de las malezas en la yuca, pone de manifiesto que en condiciones de clima y suelo de Iquitos, la variedad "Señorita" es susceptible a la competencia de malezas.

\section{CONCLUSIONES}

En parcelas de manejo de pijuayo sembradas en forma secuencial y en asociación, utilizando entre las hileras el arroz y luego de su cosecha se cultivó la yuca, los rendimientos resultaron alentadores. La localidad de siembra tiene efecto significativo sobre el comportamiento del cultivo; estas localidades de siembra tienen las mismas condiciones climáticas en el ámbito de la carretera Iquitos-Nauta; sin embargo, sus propiedades edafológicas físicos, químicos y biológicos tienen sus propias particularidades que estarían mostrando una diferencia estadística significativa $(\mathrm{p}<0,05)$, influencian en el rendimiento con promedios superiores en la localidad de El Dorado. Afirmamos que las localidades de siembra y las variedades a emplear determinan el comportamiento agronómico de los cultivos favoreciendo mejores rendimientos.

\section{AGRADECIMIENTOS}

Este trabajo fue financiado por el Programa Nacional de Innovación Agraria (PNIA), a través del proyecto "Desarrollo de un Sistema Producción Validado para Genotipos de Pijuayo Fruto en Campo de Productores Agrarios de la carretera Iquitos-Nauta en la Región Loreto", conducido por el Instituto Nacional de Innovación Agraria 
(INIA), Estación Experimental "San Roque". Los autores agradecen a Manuel Calixto Ávila Fucos por su aporte y revisión del manuscrito.

\section{REFERENCIAS BIBLIOGRÁFICAS}

Arévalo, L. 1994. Definición y clasificación de sistemas agroforestales. (http://www4. congreso.gob.pe/comisiones/1999/ciencia/ cd/inia/inia-i4/inia-i4-02.htm).

Acceso: 11/08/2020.

Arévalo, L.; Lopez, A. 1996. Yurimaguas tierra germinal. Instituto de Investigaciones de la Amazonia Peruana, Iquitos. 70pp.

Blanco, Y. 2016. El rol de las arvenses como componente en la biodiversidad de los agroecosistemas. Cultivos Tropicales, 37(4): 34-56. DOI: https://doi.org/10.13140/RG.2.2.10964. 19844

Cárdenas, R. 1999. Cultivo de yuca en la selva baja, INIA. Iquitos-Perú, 35pp.

Chibnik, 1994. Risky rivers: the economics and politics offloodplain farming in the Amazon. The University of Arizona Press, Tucson. 267pp.

Contreras, M. 2019. Implementación y transferencia tecnológica de un sistema productivo de arroz secano (Oryza sativa L.) como proyecto demostrativo, en el municipio de Achí-Bolívar. Tesis de pre-grado, Univerisdad de la Salle, Bogota. 81pp.

De Datta, S.K. 1986. Principles and practices of rice production. Wiley, New York, p. 460-467.

Fernández, E.C.M.; Serrao, E.A.S. 1992. Prototipo e modelos agrosilvipastoril sustentáives. In: Seminario Internacional sobre Meio Ambiente, Probreza e Desenvolvimiento da Amazonia (SINDAMAZONIA). Belem, PA. p. 5238-5251.

Franquet, J.; Borrás, C. 2004. Variedades $y$ mejora del arroz (Oryza sativa L.). Universitat Internacional de Catalunya, Barcelona. 454pp.
Holdridge, L.R. 1987. Ecología basada en zonas de vida. IICA, San José, Costa Rica. 216pp.

INIA, 2019. Manual de producción de arroz en suelos inundables de la región Loreto. INIA, Iquitos. 28pp.

Labarta, R.A.; White, D.; Leguía, E.; Guzmán, W.; Soto, J. 2007. La agricultura en la Amazonía ribereña del río Ucayali. ¿Una zona productiva pero poco rentable ?. Acta Amazonica, 37(2): 177-186. DOI: http://dx.doi.org/10.1590/ S0044-59672007000200002

List, G.; Laszlo, S.; Coomes, O.L. 2019. Mitigating risk for floodplain agriculture in Amazonia: a role for index-based flood insurance, Climate and Development, DOI: https://doi.org/10.108 0/17565529.2019.1674125

Martínez, G.A.; Aristizabal, Q.D.; García, R.F. 2006. Evaluación de un sistema agroforestal en callejones con productores de economía campesina del piedemonte araucano y Casanareño. CORPOICA, Villavicencio-Colombia. 45pp.

Nair, P.; Fernandes, E. 1983. Agroforestry as an alternative to shifting cultivation. In: Improved Production systems as an alternative to shifting cultivation. FAO, Rome. p. 177-190.

Noches, A. 2019. Modelo de evaluación de un cultivo de arroz para determinar su factibilidad. Tesis de pre-grado, Universidad de America, Bogotá. 67pp.

Ortiz, R.; González, R.; Ponce, M.; Fernández, C.; Martínez, J.I.; Creach, S.B.I. 2004. Importancia de la localidad en el comportamiento de variedades de soya durante siembras de primavera en cuba. Cultivos Tropicales, 25(3): 67-72.

Páez, O.E.; Medina, D.J.; Guerra, J.G.; Martínez, W.E. 1992. Las malezas y su manejo en el cultivo del arroz en Venezuela. CIAT, Cali, Colombia 174pp.

Perez-Crespo, C. 1991. Proyectos integrados de yи ca. Cali, Colombia. 261pp.

Raintree, J.B.; Warner, J. 1986. Agroforestry pathways for the intensification of shifting 
cultivation. Agroforestry Systems, 4(1): 39-54. DOI: https://doi.org/10.1007/BF01834701

Sánchez, P.A.; Benítes, J.R. 1987. Low-input cropping of soils the humid tropics selecting acid-tolerant germplasm. Science, 238: 1521-1527. DOI: https://doi.org/10.1126/ science.238.4833.1521

Scharff, S.D. 1988. Determinación del periodo crítico de competencia entre malezas y en cultivo de yuca (Manihot esculenta Crantz) Tesis de pre-grado. Universidad Nacional de la Amazonía Peruana, Iquitos. 56pp.

Suárez, L.; Mederos, V.R. 2011. Apuntes sobre el cultivo de la yuca (Manihot esculenta Crantz) tendencias actuales. Cultivos Tropicales, 32(3): 27-35.

Tamhane, R.V.; Motiramani, D.P.; Bali, Y.P. 1978. Suelos, su química y fertilidad en zonas tropicales. Ed. Diana, México, 438pp.

Trouse, A.C. 1975. Below-ground reactions in multiple cropping systems. USDA-ARS, Auburn, Alabama. 12pp.

Zuta, B.J. 1985. Efecto del tamaño de la estaca y de la modalidad de siembra en el clon de yuca "Señorita" (Manihot esculenta Crantz) Zúngaro Cocha-Iquitos. Tesis de pre-grado. Universidad Nacional de la Amazonía Peruana, Iquitos. 100pp.

Recibido: 27 de setiembre de 2020 Aceptado para publicación: 7 de diciembre de 2020 